\title{
Sonic black holes in dilute Bose-Einstein condensates
}

\author{
L. J. Garay, ${ }^{1,2}$ J. R. Anglin, ${ }^{1,3}$ J. I. Cirac, ${ }^{1}$ and P. Zoller ${ }^{1}$ \\ ${ }^{1}$ Institut für Theoretische Physik, Universität Innsbruck, Technikerstrasse 25, A-6020 Innsbruck, Austria \\ ${ }^{2}$ Instituto de Matemáticas y Física Fundamental, CSIC, C/ Serrano 121, E-28006 Madrid, Spain \\ ${ }^{3}$ Institute for Theoretical Atomic and Molecular Physics, Harvard-Smithsonian Center for Astrophysics, 60 Garden Street, \\ Cambridge, Massachusetts 02135
}

(Received 30 May 2000; published 17 January 2001)

\begin{abstract}
The sonic analog of a gravitational black hole in dilute-gas Bose-Einstein condensates is investigated. It is shown that there exist both dynamically stable and unstable configurations which, in the hydrodynamic limit, exhibit behaviors completely analogous to that of gravitational black holes. The dynamical instabilities involve the creation of quasiparticle pairs in positive and negative energy states. We illustrate these features in two qualitatively different one-dimensional models, namely, a long, thin condensate with an outcoupler laser beam providing an "atom sink," and a tight ring-shaped condensate. We also simulate the creation of a stable sonic black hole by solving the Gross-Pitaevskii equation numerically for a condensate subject to a trapping potential which is adiabatically deformed. A sonic black hole could, in this way, be created experimentally with state-of-the-art or planned technology.
\end{abstract}

DOI: 10.1103/PhysRevA.63.023611

PACS number(s): 03.75.Fi, 04.70.Dy, 04.80.-y

\section{INTRODUCTION}

Many investigations of dilute gas Bose-Einstein condensates are directed toward experimentally creating nontrivial configurations of the semiclassical mean field, or to predicting the properties of such configurations in the presence of quantum fluctuations. Such problems are hardly peculiar to condensates: the quantum neighborhoods of interesting classical backgrounds are important areas of research in most fields of physics. But ultracold dilute gases are so easy to manipulate and control, both experimentally [1] and theoretically [2], that they may allow us to decipher less amenable systems by analogy. As an essay in such an application of condensates, in this paper we discuss the theoretical framework and propose an experiment to create the analog of a black hole in the laboratory and simulate its radiative instabilities.

It is now commonly believed, even in the context of elementary particle physics, that quantum field theory arises from a still unknown underlying structure: it is an effective dynamical theory, describing the low-energy limit of collective phenomena of the underlying microscopic theory. From this viewpoint, our description of (more) fundamental phenomena, such as gravity or electromagnetism, is actually similar to the theoretical descriptions of many phenomena of condensed matter. To understand superfluidity, superconductivity, or dilute Bose-Einstein condensation, we describe the dynamics of the system in terms of collective modes (quasiparticles) whose typical size is much larger than the distances between the particles that constitute the underlying medium; but even electrons and photons must be considered as quasiparticles of a deeper theory we do not yet know. In this sense we may say that the major difference between our fundamental theories and those we use in condensed matter is that in the latter case the next microscopic level of description is actually well understood.

With this fundamental background in mind, it is not so surprising that condensed matter analogs of nontrivial con- figurations appearing in relativistic quantum field theories and gravitation can be constructed. For example, ${ }^{3} \mathrm{He}$ was proposed as a laboratory counterpart of high-energy particle physics. It was argued that, under appropriate conditions, excitations around the ground state of the system may resemble the particle spectrum of gauge theories of highenergy physics [3]. These condensed-matter systems were also used to simulate topological defects characteristic of gauge theories, and which are considered to have played a cosmological role in the early stages of the evolution of the universe such as monopoles and cosmic strings [3].

The past decade witnessed an increasing interest in simulating gravitational configurations and processes in condensed-matter systems in the laboratory. The key observation was originally made by Unruh $[4,5]$ and further analyzed by Visser [6,7]: phononic propagation in a fluid is described by a wave equation which, under appropriate conditions, can be interpreted as propagation in an effective relativistic curved space-time background, the space-time metric being entirely determined by the physical properties of the fluid under study, namely, its density and flow velocity. Unruh urged a specific motivation [4] for examining the hydrodynamic analog of an event horizon [8], namely, that as an experimentally and theoretically accessible phenomenon it might shed some light on the Hawking effect [9] (thermal radiation from black holes, stationary insofar as backreaction is negligible). In particular, one would like to gain insight into the role in the Hawking process of ultrahigh frequencies $[5,10,11]$.

An event horizon for sound waves appears in principle wherever there is a surface through which a fluid flows at the speed of sound, the flow being subsonic on one side of the surface and supersonic on the other. There is a close analogy between sound propagation on a background hydrodynamic flow, and field propagation in a curved space-time; and although hydrodynamics is only a long-wavelength effective theory for physical (super)fluids, so also field theory in curved space-time is to be considered a long-wavelength ap- 
proximation to quantum gravity [5,7]. Determining whether and how sonic black holes radiate sound, in a full calculation beyond the hydrodynamic approximation or in an actual experiment, can thus offer some suggestions about black-hole radiance and its sensitivity to high-frequency physics (beyond the Planck scale). The possibility that such high frequencies might have consequences for observably lowfrequency phenomena is one of the main reasons that black holes have deserved much attention: there is reason to expect that an event horizon can act as a microscope, giving us a view into physics on scales below the Planck length. This is because modes coming from an event horizon are redshifted into the low-energy regime as they propagate out to be observed far away from the black hole. Conversely, if we imagine tracking the observed signal back towards its source, the closer we come to the horizon the shorter the wavelength of the signal must be, until at the very horizon we must either reach infinite energy scales or encounter a breakdown in general relativity and quantum field theory in curved space-time [12].

In understanding this problem, hydrodynamic and condensed-matter analog of black holes may offer some of the experimental guidance otherwise difficult to obtain in the case of gravity [12]. Under appropriate conditions and approximations (which can be basically summarized in the requirement that the wavelengths of the perturbations be sufficiently large), the propagation of collective fluctuations (phonons) admits an effective general relativistic description, in terms of a space-time metric. This long-wavelength regime would correspond analogically to quantum field theory in curved space-time. The effective phonon metric may describe black holes, as in general relativity, and so a phonon Hawking effect may be possible; certainly the problem of arbitrarily high frequencies at the horizon is also present. But in this case, when at short wavelengths the metric approximation is no longer valid and a more microscopic theory must be used instead, the accurate microscopic theory is actually known. If the hydrodynamic system is a dilute BoseEinstein condensate, the microscopic theory is actually tractable enough that we can make reliable calculations from first principles. As we will argue, trapped bosons at ultralow temperature can indeed provide an analog to a black-hole space-time. Similar analogs were proposed in other contexts, such as superfluid helium [13], solid-state physics [14], and optics [15], but the outstanding recent experimental progress in cooling, manipulating, and controlling atoms [16] make Bose-Einstein condensates an especially powerful tool for this kind of investigation.

The basic challenge of our proposal is to keep the trapped Bose-Einstein gas sufficiently cold and well isolated to maintain a locally supersonic flow long enough to observe its intrinsic dynamics. Detecting thermal phonons radiating from the horizons would obviously be a difficult additional problem, since such radiation would be indistinguishable from many other possible heating effects. This further difficulty does not arise in our proposal, however, because the black-hole radiation we predict is not quasistationary, but grows exponentially under appropriate conditions. It should therefore be observable in the next generation of atom traps, and may also raise new issues in the theory of gravitational black holes.

In this paper, we extend and generalize the results of Ref. [17], including a more detailed analysis of the model considered there as well as a new qualitatively different case. The paper is organized as follows. In Sec. II, we show how sonic horizons in dilute Bose-Einstein condensates may appear in the hydrodynamic approximation, and discuss the regime of validity of such approximation, as well the validity of onedimensional models. Section III is devoted to the study of sonic horizons in condensates subject to tight ring-shaped external potentials. We present numerical results showing that both stable and unstable black holes may be created under realistically attainable conditions in current or nearfuture laboratories. We also study the nature of the dynamical instabilities that appear for certain configurations. In Sec. IV, we discuss a different configuration, namely, that of a sink-generated black hole in an infinite one-dimensional condensate, and show that there also exist black-hole configurations, although they are not stable. We summarize and conclude in Sec. V. The Appendix is devoted to the issues of redundancy and normalization of the dynamically unstable Bogoliubov modes (associated with complex eigenfrequencies).

\section{SONIC BLACK HOLES IN CONDENSATES}

A Bose-Einstein condensate is the ground state of a second-quantized many-body Hamiltonian for $N$ interacting bosons trapped by an external potential $V_{\text {ext }}(\mathbf{x})$ [2]. At zero temperature, when the number of atoms is large and the atomic interactions are sufficiently small, almost all the atoms are in the same single-particle quantum state $\Psi(\mathbf{x}, t)$, even if the system is slightly perturbed. The evolution of $\Psi$ is then given by the well-known Gross-Pitaevskii equation, which in appropriate units can be written as

$$
i \hbar \partial_{t} \Psi=\left(-\frac{\hbar^{2}}{2 m} \nabla^{2}+V_{\mathrm{ext}}+\frac{4 \pi a \hbar^{2}}{m}|\Psi|^{2}\right) \Psi,
$$

where $m$ is the mass of the individual atoms, and $a$ is the scattering length. The wave function of the condensate is normalized to the total number of atoms $\int d^{3} \mathbf{x}|\Psi(\mathbf{x}, t)|^{2}=N$.

Our purposes do not require solving the Gross-Pitaevskii equation with some given external potential $V_{\text {ext }}(\mathbf{x})$; our concern is the propagation of small collective perturbations of the condensate, around a background stationary state

$$
\Psi_{s}(\mathbf{x}, t)=\sqrt{\rho(\mathbf{x})} e^{i \vartheta(\mathbf{x})} e^{-i \mu t / \hbar},
$$

where $\mu$ is the chemical potential. Thus it is only necessary that it be possible, in any external potential that can be generated, to create a condensate in this state. Indeed, many realistic techniques for "quantum state engineering," to create designer potentials and bring condensates into specific states, have been proposed, and even implemented successfully [16]; our simulations indicate that currently known techniques should suffice to generate the condensate states that we propose. 
Perturbations about the stationary state $\Psi_{s}(\mathbf{x}, t)$ obey the Bogoliubov system of two coupled second-order differential equations. Within the regime of validity of the hydrodynamic (Thomas-Fermi) approximation [2], these two equations for the density perturbation $\varrho$ and the phase perturbation $\phi$ in terms of the local speed of sound

$$
c(\mathbf{x}) \equiv \frac{\hbar}{m} \sqrt{4 \pi a \rho(\mathbf{x})}
$$

and the background stationary velocity

$$
\mathbf{v} \equiv \frac{\hbar}{m} \nabla \vartheta
$$

read

$$
\dot{\varrho}=-\nabla\left(\frac{m}{4 \pi a \hbar} c^{2} \nabla \phi+\mathbf{v} \varrho\right), \quad \dot{\phi}=-\mathbf{v} \nabla \phi-\frac{4 \pi a \hbar}{m} \varrho .
$$

Furthermore, low-frequency perturbations are essentially just waves of (zero) sound. Indeed, the Bogoliubov equations may be reduced to a single second-order equation for the condensate phase perturbation $\phi$. This differential equation has the form of a relativistic wave equation $\partial_{\mu}\left(\sqrt{-g} g^{\mu \nu} \partial_{\nu} \phi\right)=0$, with $g=\operatorname{det} g_{\mu \nu}$, in an effective curved space-time with the metric $g_{\mu \nu}$ being entirely determined by the local speed of sound $c$ and the background stationary velocity $\mathbf{v}$. Up to a conformal factor, this effective metric has the form

$$
\left(g_{\mu \nu}\right)=\left(\begin{array}{cc}
-\left(c^{2}-\mathbf{v}^{2}\right) & -\mathbf{v}^{T} \\
-\mathbf{v} & \mathbf{1}
\end{array}\right)
$$

This class of metrics can possess event horizons. For instance, if an effective sink for atoms is generated at the center of a spherical trap (such as by an atom laser out-coupling technique [18]), and if the radial potential profile is suitably arranged, we can produce densities $\rho(r)$ and flow velocities $\mathbf{v}(\mathbf{x})=-v(r) \mathbf{r} / r$ such that the quantity $c^{2}-\mathbf{v}^{2}$ vanishes at a radius $r=r_{h}$, being negative inside and positive outside. The sphere at radius $r_{h}$ is a sonic event horizon completely analogous to those appearing in general relativistic black holes, in the sense that sonic perturbations cannot propagate through this surface in the outward direction $[4,5,7]$. This can be seen explicitly by writing the equation for the radial null geodesics of the metric $g_{\mu \nu}$,

$$
\dot{r}_{ \pm}=-v \pm c
$$

which can be obtained from setting the proper interval $d s^{2}$ $=g_{\mu \nu} d x^{\mu} d x^{\nu}$ equal to zero, and restricting the allowed motion to the radial direction, so that

$$
-\left(c^{2}-v^{2}\right)+2 v \dot{r}+\dot{r}^{2}=0 .
$$

The ingoing null geodesic $r_{-}(t)$ is not affected by the presence of the horizon, and can cross it in a finite coordinate time $t$. The outgoing null geodesic $r_{+}(t)$, on the other hand, needs an infinite amount of time to leave the horizon since $\dot{r}_{+}=0$ at the horizon. The physical mechanism of the sonic black hole is quite simple: inside the horizon, the background flow speed $v$ is larger than the local speed of sound $c$, and so sound waves are inexorably dragged inward.

In fact there are two conditions which must hold for this dragged sound picture to be accurate. Wavelengths larger than the black hole itself will of course not be dragged in, but merely diffracted around it. Also, perturbations must have wavelengths

$$
\lambda \gg \frac{\pi \hbar}{m c}, \quad \frac{\pi \hbar}{m c \sqrt{|1-v / c|}} .
$$

Otherwise they do not behave as sound waves, since they lie outside the regime of validity of the hydrodynamic approximation. These short-wavelength modes must be described by the full Bogoliubov equations, which allow signals to propagate faster than the local sound speed, and thus permit escape from sonic black holes. So, to identify a condensate state $\Psi_{s}$ as a sonic black hole, there must exist modes with wavelengths larger than these lower limits (which in terms of the local healing length $\xi(\mathbf{x}) \equiv \hbar /[m c(\mathbf{x})]$ read $\lambda$ $\gg 2 \pi \xi, \quad 2 \pi \xi / \sqrt{|1-v / c|}$, , but also smaller than the blackhole size. Even if such an intermediate range does exist, the modes outside it may still affect the stability of the black hole, as discussed below.

As it stands, this description is incomplete. The condensate flows continually inward, and therefore at $r=0$ there must be a sink that takes atoms out of the condensate. Otherwise, the continuity equation $\nabla(\rho \mathbf{v})=0$, which must hold for stationary configurations, will be violated. From a physical point of view, such a sink can be accomplished by means of an outcoupler laser beam at the origin. (Such outcouplers are the basic mechanisms for making trapped condensates into "'atom lasers," and they were already demonstrated experimentally by several groups. A tightly focused laser pulse changes the internal state of the atoms at a particular point in the trap, and can also be made to give them a large momentum impulse. This ejects them so rapidly through the always dilute condensate cloud that they do not significantly disturb it; effectively, they simply disappear.)

We analyzed several specific systems which may be suitable theoretical models for future experiments, and found that the qualitative behavior is analogous in all of them. Black holes which require atom sinks are both theoretically and experimentally more involved, however; moreover, maintaining a steady transonic flow into a sink may require either a very large condensate or some means of replenishment. We will therefore first discuss an alternative configuration which may be experimentally more accessible, and whose description is particularly simple: a condensate in a very thin ring that effectively behaves as a periodic onedimensional system (Fig. 1). Under conditions that we will discuss, the supersonic region in a ring may be bounded by two horizons: a black-hole horizon through which phonons cannot exit, and a "white-hole" horizon through which they cannot enter. Then we will analyze another simple one- 


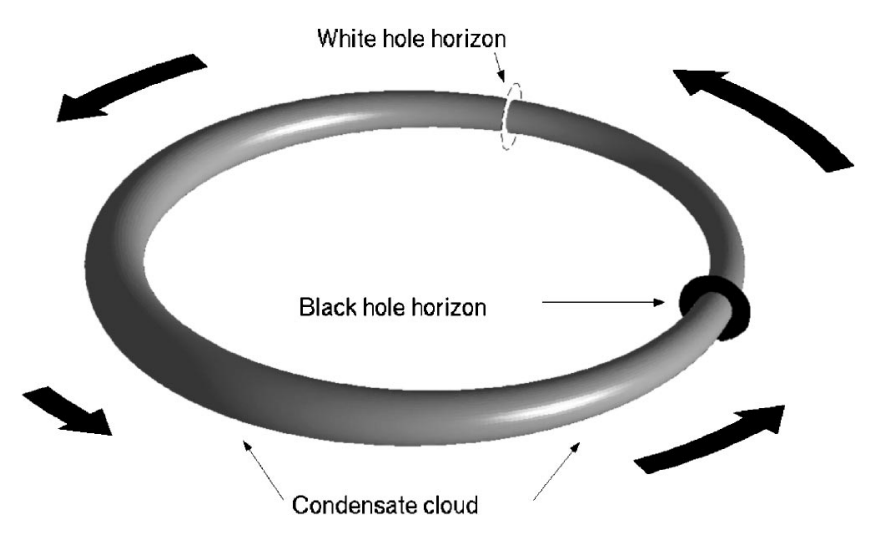

FIG. 1. The tight ring-shaped configuration, with both black and white horizons, and no singularity. Arrows indicate condensate flow velocity, with longer arrows for faster flow.

dimensional model, of a long, straight condensate with an atom sink at the center (Fig. 2).

The existence of instabilities that do not show up in the one-dimensional approximation is an important question in condensate physics, which is under active theoretical and experimental investigation. The essential principles have long been clear, inasmuch as the current dilute condensates really are the weakly interacting Bose gases that have been used as toy models for superfluidity for several decades. The fact that actual critical velocities in liquid helium are generally far below the Landau critical velocity is understood to be due partly to the roton feature of the helium dispersion relation, but this is not present in the dilute condensates. Viscosity also arises due to surface effects, however, and these may indeed afflict dilute condensates as well. The point here is that in addition to the bulk phonon modes considered by Landau, and quite adequately represented in our onedimensional analysis, there may in principle be surface modes, with a different (and generally lower) dispersion curve. If such modes exist and are unstable, it is very often the case that, as they grow beyond the perturbative regime, they turn into quantized vortices, which can cut through the supercurrent and so lower it.

Whether or not such unstable surface modes actually exist in the Bogoliubov spectrum of a dilute condensate is an issue

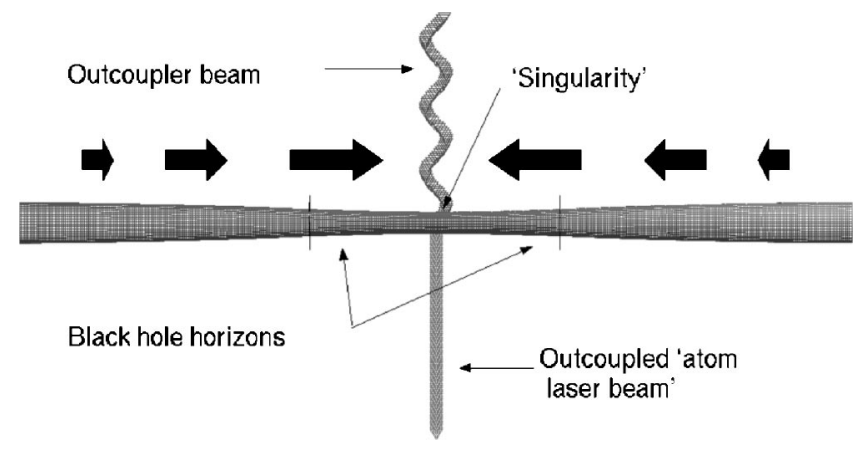

FIG. 2. The tight cigar-shaped configuration, with two blackhole horizons and a "singularity" where the condensate is outcoupled. Arrows indicate the condensate flow velocity, with longer arrows for faster flow. that was recently analyzed both numerically and analytically, and it is quite clear that such surface modes exist only if the confining potential is quite rough (which is not only easy to avoid with a magnetic or optical trapping field, but very hard to achieve) [19], or if the condensate dynamics in the directions perpendicular to the flow is hydrodynamic. That is, the condensate must be at least a few healing lengths thick, so that surface modes decaying on the healing length scale can satisfy all the required boundary conditions [20]. By saying that we are considering an effectively one-dimensional condensate, we mean precisely that this is not the case. For instance, for the tight-ring model, in this regime, the radial trap scale is the shortest length scale in the problem, and the radial trap frequency is the highest frequency; this effectively means that excitations of nontrivial radial modes, including surface modes, are energetically frozen out. (In the limit of radial confinement within the scattering length, our model breaks down for other reasons-but the scattering length can easily be two orders of magnitude smaller than the healing length.) The issue of the supercurrent stability in tightly confined ring shaped traps was addressed in Ref. [21], where the authors arrived at a positive conclusion and also clarified the role of finite temperature and possible trap anisotropy.

\section{SONIC BLACK/WHITE HOLES IN A RING}

In a sufficiently tight ring-shaped external potential of radius $R$, motion in radial $(r)$ and axial $(z)$ cylindrical coordinates is effectively frozen. We can then write the wave function as $\Psi(z, r, \theta, \tau)=f(z, r) \Phi(\theta, \tau)$, and normalize $\Phi$ to the number of atoms in the condensate $\int_{0}^{2 \pi} d \theta|\Phi(\theta)|^{2}=N$, where with the azimuthal coordinate $\theta$ we have introduced the dimensionless time $\tau=\left(\hbar / m R^{2}\right) t$. The Gross-Pitaevskii equation thus becomes effectively one-dimensional,

$$
i \partial_{\tau} \Phi=\left(-\frac{1}{2} \partial_{\theta}^{2}+\mathcal{V}_{\text {ext }}+\frac{\mathcal{U}}{N}|\Phi|^{2}\right) \Phi
$$

where $\mathcal{U} \equiv 4 \pi a N R^{2} \int d z d r r|f(z, r)|^{4}$, and $\mathcal{V}_{\text {ext }}(\theta)$ is the dimensionless effective potential (in which we have already included the chemical potential) that results from the dimensional reduction. The stationary solution can then be written as $\Phi_{s}(\theta, \tau)=\sqrt{\rho(\theta)} e^{i \int d \theta v(\theta)}$, and the local dimensionless angular speed of sound as $c(\theta)=\sqrt{\mathcal{U} \rho(\theta) / N}$. Periodic boundary conditions around the ring require the "winding number' ' $w \equiv(1 / 2 \pi) \int_{0}^{2 \pi} d \theta v(\theta)$ to be an integer.

The qualitative behavior of horizons in this system is well represented by the two-parameter family of condensate densities,

$$
\rho(\theta)=\frac{N}{2 \pi}(1+b \cos \theta),
$$

where $b \in[0,1]$. Continuity, $\partial_{\theta}(\rho v)=0$, then determines the dimensionless flow-velocity field

$$
v(\theta)=\frac{\mathcal{U} w \sqrt{1-b^{2}}}{2 \pi c(\theta)^{2}},
$$


which depends on $w$ as a third discrete independent parameter. Requiring that $\Phi_{s}(\theta, \tau)$ be a stationary solution to the Gross-Pitaevskii equation then determines how the trapping potential must be modulated as a function of $\theta$. All the properties of the condensate, including whether and where it has sonic horizons, and whether or not they are stable, are thus functions of $\mathcal{U}, b$, and $w$. For instance, if we require that the horizons be located at $\theta_{h}= \pm \pi / 2$, which imposes the relation $\mathcal{U}=2 \pi w^{2}\left(1-b^{2}\right)$, then we must have $c^{2}-v^{2}$ positive for $\theta \in(-\pi / 2, \pi / 2)$, zero at $\theta_{h}= \pm \pi / 2$, and negative otherwise, provided that $\mathcal{U}<2 \pi w^{2}$. The further requirement that perturbations on wavelengths shorter than the inner and the outer regions are indeed phononic implies $\mathcal{U} \gg 2 \pi$, which in turn requires $w \gg 1$ and $1 \gg b \gg 1 / w^{2}$. In fact, a detailed analysis shows that $w \geqslant 5$ is sufficient.

\section{A. Stability}

The mere existence of a black-hole solution does not necessarily mean that it is physically realizable: it should also be stable over sufficiently long-time scales. Since stability must be checked for perturbations on all wavelengths, the full Bogoliubov [2] spectrum must be determined. For large black holes within large, slowly varying condensates, this Bogoliubov problem may be solved using WKB methods that closely resemble those used for solving relativistic field theories in true black-hole space-times [11]. A detailed adaptation of these methods to the Bogoliubov problem will be presented elsewhere [22]. The results are qualitatively similar to those we found for black holes in finite traps with low winding number, where we resorted to numerical methods because, in these cases, WKB techniques may fail for just those modes which threaten to be unstable.

Our numerical approach for our three-parameter family of black/white holes in the ring-shaped condensate has been to write the Bogoliubov equations in discrete Fourier space, and then truncate the resulting infinite-dimensional eigenvalue problem. Indeed, writing the wave funtion as $\Phi=\Phi_{s}$ $+\varphi e^{i \int d \theta v(\theta)}$, decomposing the perturbation $\varphi$ in discrete modes

$$
\begin{aligned}
\varphi(\theta, \tau)= & \sum_{\omega, n} e^{-i \omega \tau} e^{i n \theta} A_{\omega, n} u_{\omega, n}(\theta) \\
& +e^{i \omega^{*} \tau} e^{-i n \theta} A_{\omega, n}^{*} v_{\omega, n}^{*}(\theta),
\end{aligned}
$$

and substituting into the Gross-Pitaevskii equation, we obtain the following equation for the modes $u_{\omega, n}$ and $v_{\omega, n}$ :

$$
\omega\left(\begin{array}{c}
u_{\omega, n} \\
v_{\omega, n}
\end{array}\right)=\sum_{p}\left(\begin{array}{cc}
h_{n p}^{+} & f_{n p} \\
-f_{n p} & h_{n p}^{-}
\end{array}\right)\left(\begin{array}{l}
u_{\omega, p} \\
v_{\omega, p}
\end{array}\right) .
$$

In this equation,

$$
f_{n p}=\frac{1}{2 \pi} \int_{0}^{2 \pi} d \theta e^{-i(n-p) \theta} c(\theta)^{2},
$$

$$
\begin{aligned}
h_{n p}^{ \pm}= & \pm \frac{n^{2}}{2} \delta_{n p}+\frac{1}{2 \pi} \int_{0}^{2 \pi} d \theta e^{-i(n-p) \theta} \\
& \times\left[p v(\theta)-\frac{1}{2} v^{\prime}(\theta) \pm\left(c(\theta)^{2}+\frac{1}{2} \frac{c^{\prime \prime}(\theta)}{c(\theta)}\right)\right],
\end{aligned}
$$

which, after some lengthy calculations, can be written as

$$
\begin{aligned}
f_{n p} & =\frac{\mathcal{U}}{2 \pi}\left(\delta_{n, p}+\frac{b}{2} \delta_{n, p+1}+\frac{b}{2} \delta_{n, p-1}\right), \\
h_{n p}^{ \pm}= & \frac{1}{2}(n+p) w \sqrt{1-b^{2}} \alpha_{n-p} \\
& \pm\left(f_{n p}+\frac{4 n^{2}-1}{8} \delta_{n, p}+\frac{1-b^{2}}{8} \beta_{n-p}\right),
\end{aligned}
$$

where

$$
\begin{gathered}
\alpha_{i}=\sum_{j \geqslant|i|, i+j \text { even }}^{\infty}\left(\frac{-b}{2}\right)^{j}\left(\begin{array}{c}
j \\
(i+j) / 2
\end{array}\right), \\
\beta_{i}=\sum_{j \geqslant|i|, i+j \text { even }}^{\infty}\left(\frac{-b}{2}\right)^{j}\left(\begin{array}{c}
j \\
(i+j) / 2
\end{array}\right)(j+1) .
\end{gathered}
$$

Eliminating Fourier components above a sufficiently high cutoff $Q$ has a negligible effect on possible instabilities, which can be shown to occur at relatively long wavelengths. We then face an eigenvalue problem for the $2(Q+1)$ $\times 2(Q+1)$ matrix built out of blocks of the form

$$
\left(\begin{array}{cc}
h_{n p}^{+} & f_{n p} \\
-f_{n p} & h_{n p}^{-}
\end{array}\right)
$$

The numerical solution to this eigenvalue equation, together with the normalization condition $\int d \theta\left(u_{\omega^{*}, n}^{*} u_{\omega^{\prime}, n^{\prime}}\right.$ $\left.-v_{\omega^{*}, n}^{*} v_{\omega^{\prime}, n^{\prime}}\right)=\delta_{n n^{\prime}} \delta_{\omega \omega^{\prime}}$, provides the allowed frequencies. Real negative eigenfrequencies for modes of positive norm are always present, which means that black-hole configurations are energetically unstable, as expected. This feature is inherent in supersonic flow, since the speed of sound is also the Landau critical velocity. In a sufficiently cold and dilute condensate, however, the time scale for dissipation may in principle be made very long, and so these energetic instabilities need not be problematic [23].

More serious are dynamical instabilities, which occur for modes with complex eigenfrequencies. Since the Bogoliubov theory is based on a quantized Hamiltonian that is Hermitian, there are certainly no complex energy eigenvalues; but the natural frequencies of normal modes can indeed be complex [in which case the usual rule, that energy eigenvalues are $\hbar(n+1 / 2)$ times the mode frequencies, simply breaks down]. A detailed discussion of the quantum mechanics of dynamical instability is presented in the Appendix; for the purposes of our main discussion it suffices to note that complex (mode) eigenfrequencies are indeed genuine physical phenomena, and by no means a numerical artifact. For suffi- 


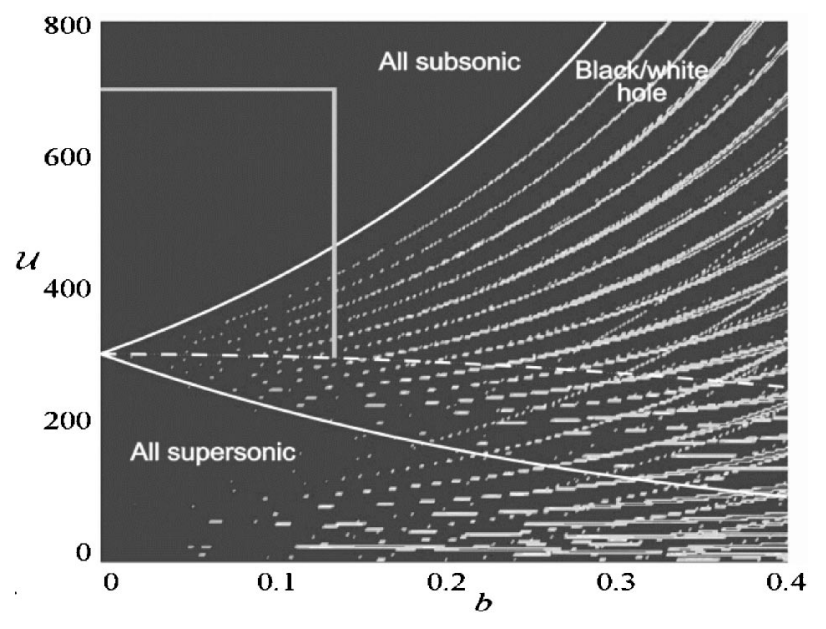

FIG. 3. Stability diagram for winding number $w=7$. Solid darkgray areas represent the regions of stability. Smaller plots at higher resolution confirm that the unstable "fingers" are actually smooth and unbroken. Points on the dashed curve are states with horizons at $\theta_{h}= \pm \pi / 2$, so that the black/white hole fills half the ring.

cently high values of the cutoff (e.g., $Q \geqslant 25$ in our calculations), the complex eigenfrequencies obtained from the truncated eigenvalue problem become independent of the cutoff within the numerical error. The existence and rapidity of dynamical instabilities depend sensitively on $(\mathcal{U}, b, w)$. For instance, see Fig. 3 for a contour plot of the maximum of the absolute values of the imaginary part of all eigenfrequencies for $w=7$, showing that the regions of instability are long, thin fingers in the $(\mathcal{U}, b)$ plane. Figure 4 shows the size of the largest absolute value of the instabilities for each point on the dashed curve of Fig. 3. It illustrates the important fact that the size of the imaginary parts, which gives the rate of the instabilities, increases starting from zero, quite rapidly with $b$, although they remain small as compared with the real parts.

\section{B. Creation of a black/white hole}

The stability diagram of Fig. 3 suggests a strategy for creating a sonic black hole from an initial stable state. Within the upper subsonic region, the vertical axis $b=0$ corresponds to a homogeneous persistent current in a ring, which can in principle be created using different techniques [24]. Gradually changing $\mathcal{U}$ and $b$, it is possible to move from such an initial state to a black/white hole state, along a path lying

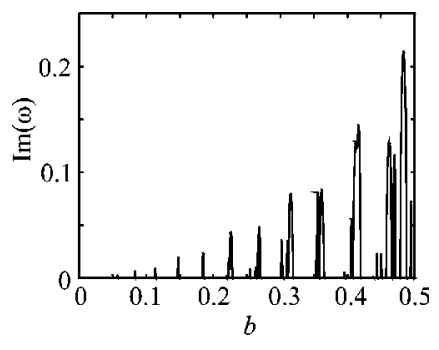

FIG. 4. Stability digram for black/white holes of maximum size, i.e., along the dashed line of Fig. 3.

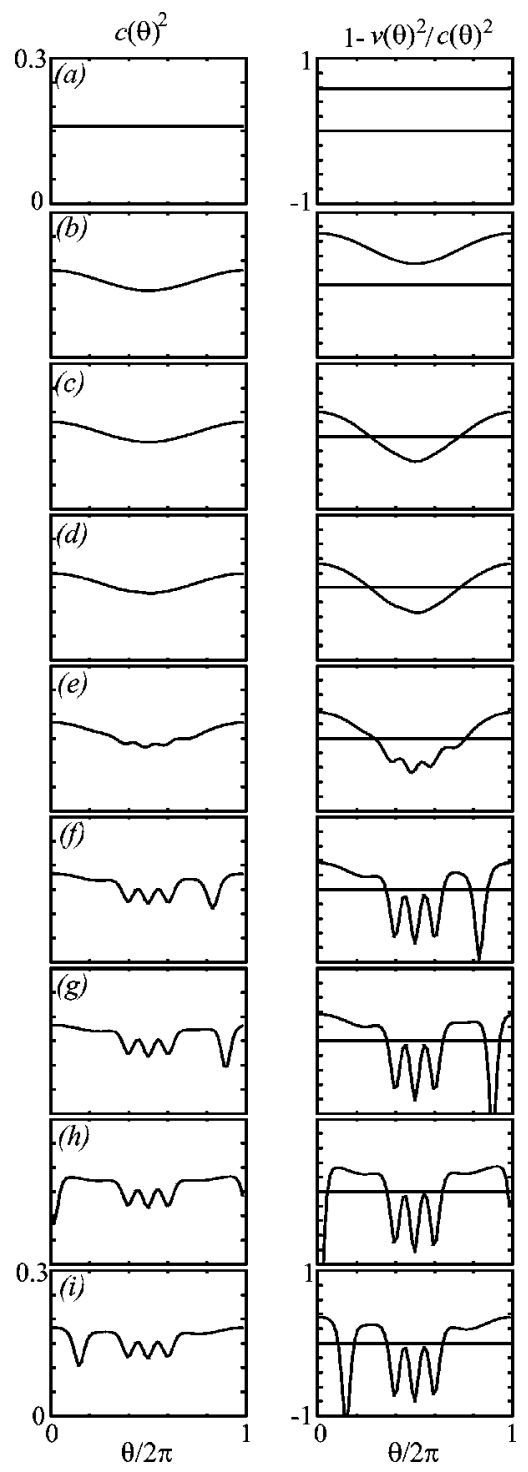

FIG. 5. Simulation of creation of a stable black/white hole, and subsequent evolution into an unstable region. (a)-(d) are snapshots taken at the initial time (a); at an intermediate time, still within the subsonic region (b); when black/white holes of maximum size are approached (c); and after a long time in that configuration (d). Then the parameters are changed along the dashed curve of Fig. 3 to enter an unstable region (e) and kept there (f)-(i). It can be observed that a perturbation grows at the black-hole horizon, and travels rightward until it enters the white-hole horizon.

almost entirely within the stable region, and only passing briefly through instabilities where they are sufficiently small to cause no difficulty.

Indeed, we have simulated this process of adiabatic creation of a sonic black/white hole by solving numerically (using the split operator method) the time-dependent GrossPitaevskii equation (1) that provides the evolution of the condensate when the parameters of the trapping potential change so as to move the condensate state along various paths in parameter space. One of these paths is shown in Fig. 3 (light-gray solid line): we start with a current at $w=7$ and $b=0$, and sufficiently high $\mathcal{U}$ [Fig. 5(a)]; we then increase $b$ 
adiabatically keeping $\mathcal{U}$ fixed until an appropriate value is reached [Fig. 5(b)]; finally, keeping $b$ constant, we decrease $\mathcal{U}$ adiabatically (which can be physically implemented by decreasing the radius of the ring trap), until we meet the dashed contour for black holes of comfortable size [Fig. 5(c)]. Our simulations confirm that the small instabilities which briefly appear in the process of creation do not disrupt the adiabatic evolution. The final quantum state of the condensate, obtained by this procedure, indeed represents a stable black/white hole. We have further checked the stability of this final configuration by numerically solving the Gross-Pitaevskii equation (1) for very long periods of time (as compared with any characteristic time scale of the condensate) and for fixed values of the trap parameters. This evolution reflects the fact that no imaginary frequencies are present, as predicted from the mode analysis, and that the final state is indeed stationary [Fig. 5(d)]. Once the black/ white hole has been created, one could further change the parameters $(\mathcal{U}, b)$ so as to move between the unstable "fingers" into a stable region of higher $b$ (a deeper hole).

\section{Quasiparticle pair creation}

Instead of navigating the stable region of parameter space, one could deliberately enter an unstable region [Figs. 5(e)5(i)]. In this case, the black hole should disappear in an explosion of phonons, which may be easy to detect experimentally. Such an event might be related to the evaporation process suggested for real black holes, in the sense that pairs of quasiparticles are created near the horizon in both positive and negative energy modes. We will explain this point briefly; a more detailed exposition is included in the Appendix.

In the language of second quantization, the perturbation field operator $\varphi$ satisfies the linear equation

$$
i \dot{\varphi}=-\frac{1}{2} \varphi^{\prime \prime}-i v \varphi^{\prime}+\left(\frac{1}{2} \frac{c^{\prime \prime}}{c}-\frac{i}{2} v^{\prime}+c^{2}\right) \varphi+c^{2} \varphi^{\dagger},
$$

which, taking into account that $\left[\varphi(\theta), \varphi^{\dagger}\left(\theta^{\prime}\right)\right]=\delta\left(\theta-\theta^{\prime}\right)$, can be written as

$$
i \dot{\varphi}=[\varphi, H],
$$

where the Bogoliubov Hamiltonian is

$$
\begin{aligned}
H= & \int d \theta\left[-\frac{1}{2} \varphi^{\dagger} \varphi^{\prime \prime}-i v \varphi^{\dagger} \varphi^{\prime}+\left(\frac{1}{2} \frac{c^{\prime \prime}}{c}-\frac{i}{2} v^{\prime}+c^{2}\right) \varphi^{\dagger} \varphi\right. \\
& \left.+\frac{1}{2}\left(\varphi^{\dagger} \varphi^{\dagger}+\varphi \varphi\right)\right] .
\end{aligned}
$$

The Hermiticity of the Bogoliubov linearized Hamiltonian implies that eigenmodes with complex frequencies always appear in dual pairs, whose frequencies are complex conjugate. In the language of second quantization, the linearized Hamiltonian for each such pair has the form

$$
H=\sum_{n}\left(\omega A_{\omega *, n}^{\dagger} A_{\omega, n}+\omega^{*} A_{\omega, n}^{\dagger} A_{\omega^{*}, n}\right),
$$

and the only nonvanishing commutators among these operators are $\left[A_{\omega, n}, A_{\omega^{*}, n^{\prime}}^{\dagger}\right]=\delta_{n n^{\prime}}$. The asterisk on the subscript is important: the mode with frequency $\omega^{*}$ is a different mode from the one with frequency $\omega$, and $A_{\omega^{*}, n}^{\dagger}$ is not the Hermitian conjugate of $A_{\omega, n}^{\dagger}$ ! It is therefore clear that none of these operators is actually a harmonic oscillator creation or annihilation operator in the usual sense. However, the linear combinations

$$
a_{n}=\frac{1}{\sqrt{2}}\left(A_{\omega, n}+A_{\omega *, n}\right), \quad b_{n}=\frac{i}{\sqrt{2}}\left(A_{\omega, n}^{\dagger}+A_{\omega *, n}^{\dagger}\right),
$$

and their Hermitian conjugates, are true annhilation and creation operators, with the standard commutation relations, and in terms of these the Bogoliubov Hamiltonian becomes

$$
H=\sum_{n}\left[\operatorname{Re}(\omega)\left(a_{n}^{\dagger} a_{n}-b_{n}^{\dagger} b_{n}\right)-\operatorname{Im}(\omega)\left(a_{n}^{\dagger} b_{n}^{\dagger}+a_{n} b_{n}\right)\right] .
$$

This interaction obviously leads to self-amplifying creation of positive and negative frequency pairs. Evaporation through an exponentially self-amplifying instability is not equivalent, however, to the usual kind of Hawking radiation [11]; this issue will be discussed in detail elsewhere.

\section{SINK-GENERATED BLACK HOLES}

Condensates which develop black-hole behaviors by means of flows generated by laser-driven sinks also present regions of stability and instability in parameter space and, in this sense, their behavior is analogous to that in a ringshaped trapping potential. Here we present a simple model that exhibits the main qualitative features of more general situations and that can be studied analytically. Although in this model we study a condensate of infinite size, in more realistic models or experiments it will suffice to take condensates which are sufficiently large, since the stability pattern is not significantly affected by the (large but finite) size of the condensate.

\section{A. Model}

Let us consider a tight cigar-shaped condensate of infinite size such that the motion in the $(y, z)$ plane is effectively frozen. In appropriate dimensionless units, the effectively one-dimensional Gross-Pitaevskii equation thus becomes

$$
i \partial_{\tau} \Phi=\left(-\frac{1}{2} \partial_{x}^{2}+\mathcal{V}_{\mathrm{ext}}+\mathcal{U}|\Phi|^{2}\right) \Phi,
$$

with the normalization condition

$$
\lim _{D \rightarrow \infty} \frac{1}{2 D} \int_{-D}^{D} d x|\Phi(x)|^{2}=n
$$

In this equation, $\mathcal{V}_{\text {ext }}$ is the dimensionless effective potential that results form the dimensional reduction, which already includes the chemical potential. 
In order to obtain a black hole configuration, let us choose the potential $\mathcal{V}_{\text {ext }}$ so that it produces a profile for the speed of sound $c(x)=\sqrt{\mathcal{U} \rho(x)}$ of the form

$$
c(x)=\left\{\begin{array}{l}
c_{0}, \quad|x|<L \\
c_{0}[1+(\sigma-1) x / \epsilon], \quad L<|x|<L+\epsilon \\
\sigma c_{0}, \quad L+\epsilon<|x|,
\end{array}\right.
$$

with $\sigma>1$, and a flow velocity in the inward direction. The continuity equation then provides the flow velocity profile

$$
v(x)=-\frac{v_{0} c_{0}^{2}}{c(x)^{2}} \frac{x}{|x|},
$$

where $v_{0}$ is the absolute value of the flow velocity in the inner region.

As it stands, this model fails to fulfill the continuity equation at $x=0$. In order to take this into account, we will also introduce a sink of atoms at $x=0$ that takes atoms out of the condensate (this can be physically implemented by means of a laser). From a mathematical point of view, this can be modeled by an additional term in the equation of the form $-i E \delta(x)$, which indeed induces loss of atoms at $x=0$. Equivalently, it can be represented by boundary conditions of the form

$$
\begin{gathered}
\Phi\left(0^{+}, \tau\right)-\Phi\left(0^{-}, \tau\right)=0, \\
\Phi^{\prime}\left(0^{+}, \tau\right)-\Phi^{\prime}\left(0^{-}, \tau\right)=-2 i E \Phi(0, \tau),
\end{gathered}
$$

which determine the flow velocity inside in terms of the characteristics of the outcoupler laser, namely, $v_{0}=E$.

Perturbations $\phi$ around this stationary state $\Phi_{s}$ $=\sqrt{\rho} e^{i \int v(x) d x}$, such that $\Phi=\Phi_{s}+\phi$ (note that for convenience we have chosen a different convention as compared with the ring in which $\Phi=\Phi_{s}+\varphi e^{i{ }^{i} v}$ ), must satisfy boundary conditions (3) and the equation

$$
i \dot{\phi}=-\frac{1}{2} \phi^{\prime \prime}+\left(c^{2}-v^{2} / 2+c^{\prime \prime} / 2 c\right) \phi+c^{2} e^{2 i \int^{x} v} \phi^{*},
$$

where

$$
\frac{c^{\prime \prime}}{c}=\frac{\sigma-1}{\epsilon}\left[\delta(|x|-L)-\frac{1}{\sigma} \delta(|x|-L-\epsilon)\right] .
$$

As a further simplifying assumption, we will assume that $v_{0} \epsilon \ll 1$, so that

$$
\left|\int_{L}^{x} d x^{\prime} v\left(x^{\prime}\right)\right| \leqslant \int_{L}^{L+\epsilon} d x \frac{v_{0}}{[1+(\sigma-1) x / \epsilon]^{2}} \leqslant v_{0} \epsilon \ll 1 .
$$

Let us now expand the perturbation $\phi$ in modes

$$
\phi=\sum_{\omega, k}\left[A_{\omega, k} u_{\omega, k}(x) e^{-i \omega \tau}+A_{\omega, k}^{*} v_{\omega, k}(x)^{*} e^{i \omega^{*} \tau}\right] .
$$

Then, the modes $u_{\omega, k}(x)$ and $v_{\omega, k}(x)$ satisfy, in each region, the Bogoliubov equations

$$
\begin{gathered}
\omega u_{\omega, k}=-\frac{1}{2} u_{\omega, k}^{\prime \prime}+\left(c^{2}-v^{2} / 2\right) u_{\omega, k}+c^{2} e^{2 i \int^{x} v} v_{\omega, k}, \\
\omega v_{\omega, k}=\frac{1}{2} v_{\omega, k}^{\prime \prime}-\left(c^{2}-v^{2} / 2\right) v_{\omega, k}-c^{2} e^{-2 i \int^{x} v} u_{\omega, k} .
\end{gathered}
$$

\section{B. Matching conditions}

The intermediate regions $L<|x|<L+\epsilon$ provide a connection between the perturbation modes in the inner and outer regions. Once these connection formulas have been established, in the limit of small $\epsilon$, we will only need to study the inside and outside modes and their relation through such formulas. The case of an abrupt horizon, in which the background condensate velocity is steeply and linearly ramped within a very short interval, is obviously quite special, and it does not particularly resemble the horizon of a large black hole in Einsteinian gravity. But the connection formula that we derive for this case will qualitatively resemble those that are obtained, with considerably more technical effort, for smoother horizons [22]. In addition, the results we will obtain for the global Bogoliubov spectrum of the condensate black hole will indeed be representative of more generic cases.

In the intermediate regions, $L<|x|<L+\epsilon$, the factors $e^{ \pm 2 i \int^{x} v}$ in the last terms of Eqs. (4) become $1+O(\epsilon)$. Then the solutions of these equations are

$$
\begin{aligned}
& u_{\omega, k}=\alpha_{\omega, k}+\beta_{\omega, k} x / \epsilon+O\left(\epsilon^{2}\right), \\
& v_{\omega, k}=\gamma_{\omega, k}+\kappa_{\omega, k} x / \epsilon+O\left(\epsilon^{2}\right),
\end{aligned}
$$

as can be easily seen by defining the variable $q=x / \epsilon$ so that the equations become

$$
\partial_{q}^{2} u_{\omega, k}=\partial_{q}^{2} v_{\omega, k}=O\left(\epsilon^{2}\right) .
$$

The singular character of $c^{\prime \prime} / c$ at $|x|=L, L+\epsilon$ can be substituted by matching conditions at $|x|=L, L+\epsilon$, which will in turn provide the connection formulas between the modes outside $(|x|>L)$ and the modes inside $(|x|<L)$. Furthermore, the symmetry of the problem allows us to study the region $x>0$.

These matching conditions are

$$
\begin{gathered}
\phi\left(L^{+}\right)-\phi\left(L^{-}\right)=0, \\
\phi^{\prime}\left(L^{+}\right)-\phi^{\prime}\left(L^{-}\right)=\frac{\sigma-1}{\epsilon} \phi(L), \\
\phi\left(L+\epsilon^{+}\right)-\phi\left(L+\epsilon^{-}\right)=0, \\
\phi^{\prime}\left(L+\epsilon^{+}\right)-\phi^{\prime}\left(L+\epsilon^{-}\right)=-\frac{\sigma-1}{\sigma \epsilon} \phi(L+\epsilon) .
\end{gathered}
$$

These equations, together with the form of the modes in the region $L<x<L+\epsilon$, provide the connection formulas between the inside and outside modes (from now on we will drop the subindex $\omega$ ) 


$$
\begin{gathered}
u_{\mathrm{in}, k}(L)=-\epsilon u_{\mathrm{out}, k}^{\prime}(L)+\frac{1}{\sigma} u_{\mathrm{out}, k}(L), \\
u_{\mathrm{in}, k}^{\prime}(L)=\sigma u_{\mathrm{out}, k}^{\prime}(L),
\end{gathered}
$$

and likewise for the modes $v_{\text {in,out }}$.

\section{Dispersion relation and boundary conditions for large $x$}

In each of the regions (inside and outside), we can write

$$
u_{k}(x)=u_{k} e^{i(k-|v|)(x-L)}, \quad v_{k}(x)=v_{k} e^{i(k+|v|)(x-L)} .
$$

Upon substitution of this expansion into the Bogoliubov equations (4), we obtain, for each region, the set of algebraic equations

$$
h_{k}^{-} u_{k}+c^{2} v_{k}=0, \quad c^{2} u_{k}+h_{k}^{+} v_{k}=0
$$

where $h_{k}^{ \pm}=k^{2} / 2+c^{2} \pm(k|v|+\omega)$. For these equations to have a solution, the determinant must vanish, thus providing the dispersion relation

$$
k^{4} / 4+\left(c^{2}-v^{2}\right) k^{2}-2 \omega|v| k-\omega^{2}=0,
$$

which, for fixed $\omega$, is a fourth-order equation for $k$. For each of the four solutions, $u_{k}$ and $v_{k}$ must be related by

$$
v_{k}=h_{k} u_{k} \quad \text { with } \quad h_{k}=-\frac{1}{c^{2}}\left(k^{2} / 2+c^{2}-k|v|-\omega\right) \text {. }
$$

The constant coefficients $u_{k}$ can be regarded as normalization constants, and will be set to unity. Let us study the possible solutions to the dispersion relation depending on whether $\omega$ is real or complex.

Complex frequencies. In this case all four solutions are pure complex, two of them with positive imaginary parts and two of them with negative imaginary parts.

In order to prove this statement, let us first assume that there exists a real solution $k$ for a complex $\omega=\varpi+i \gamma$. Then the imaginary part of Eq. (6) implies that $\varpi=-k|v|$. Introducing this result into its real part leads to $\gamma^{2}=-\left(k^{4} / 4\right.$ $+c^{2} k^{2}$ ) which is impossible to fulfill because $k$ is real. So the four solutions are complex.

Because of continuity, all $\omega$ in the upper-half complex $\omega$ plane have the same number of solutions with positive imaginary part. Otherwise, for some $\omega$ 's there should exist a real solution that interpolates between positive and negative imaginary part solutions; however, this is not possible, as we have seen.

Now let us concentrate on small frequencies, i.e., on frequencies around $\omega=0$. For $\omega=0$, we have a double root at $k=0$. The other two solutions are $k= \pm 2 \sqrt{v^{2}-c^{2}}$, which are real for $c^{2}<v^{2}$ (i.e., inside) and pure imaginary for $c^{2}>v^{2}$ (i.e., outside). Let us follow these four solutions when $\omega$ $=i \varepsilon$. The solutions coming from the double root $k=0$ will now be of the form $k=k_{r}+i \varepsilon k_{i}$. It is easy to see that $k_{r}$ $=0$ and $k_{i}=1 /(-|v| \pm c)$. If $c>|v|$, one is positive and one is negative. If $c<|v|$, both of them are negative. On the other hand, the solutions $k= \pm 2 \sqrt{v^{2}-c^{2}}$, for $c^{2}>v^{2}$, are already complex conjugate. For $c^{2}<v^{2}$, we write $k=k_{r}+i \varepsilon k_{i}$ and introduce it into Eq. (6). We then see that at first order in $\varepsilon$, $k_{i}=|v| /\left(v^{2}-c^{2}\right)>0$. Thus we have seen that for $\omega=i \varepsilon$, we have two solutions with positive imaginary part and two with negative parts in any case (inside and outside). But if this is so for $\omega=i \varepsilon$ it must be true in the whole upper $\omega$ plane and consequently in the whole complex $\omega$ plane. In the inside region all possible solutions are in principle allowed but outside we are only left with the two that have $\operatorname{Im}(k)>0$, because the other two grow exponentially.

Real frequencies. Outside $\left(c^{2}>v^{2}\right)$, there are two real and two complex conjugate solutions. Of these two complex solutions, only one is allowed [the one with $\operatorname{Im}(k)>0$ ] because the other grows exponentially. Inside $\left(c^{2}<v^{2}\right)$, for $\omega$ $>\omega_{\max }$, there are two real and two complex conjugate solutions; for $\omega<\omega_{\max }$ there are four real solutions; the value $\omega=\omega_{\max }$ is a bifurcating point.

\section{Connection formulas for complex frequencies}

Since we are interested in the existence of dynamical instabilities, we will concentrate on the case in which $\omega$ is complex. Then, as we have seen, the dispersion equation (6) has four complex solutions for $k$ in each region. Inside, all four solutions $k_{\mathrm{in}, i}, i=1, \ldots, 4$ are in principle possible, but outside those with $\operatorname{Im}\left(k_{\text {out }}\right)<0$ will increase exponentially. Therefore, up to corrections coming from the finite size of the condensate, which we ignore here, only modes associated with $k_{\text {out }, \alpha}, \alpha=1,2$, such that $\operatorname{Im}\left(k_{\text {out }, \alpha}\right)>0$, are allowed. Each mode $u_{\text {out, } \alpha}(x)=e^{i\left(k_{\text {out }, \alpha}-v_{0} / \sigma^{2}\right)(x-L)}$ will match a linear combination $u_{\mathrm{in}, \alpha}(x)=\sum_{i} F_{\alpha i} u_{\mathrm{in}, i}(x)$ of modes $u_{\mathrm{in}, i}(x)$ inside, i.e.,

$$
u_{\mathrm{in}, \alpha}(x)=\sum_{i} F_{\alpha i} e^{i\left(k_{\mathrm{in}, i}-v_{0}\right)(x-L)}
$$

and similarly for $v_{\text {out }, \alpha}$ and $v_{\text {in, } \alpha}$,

$$
v_{\mathrm{in}, \alpha}(x)=\sum_{i} F_{\alpha i} h_{\mathrm{in}, i} e^{i\left(k_{\mathrm{in}, i}+v_{0}\right)(x-L)} .
$$

After some straightforward calculations, it can be seen that these connecting coefficients $F_{\alpha i}$ are given by $F_{\alpha i}$ $=\Sigma_{j}\left(M^{-1}\right)_{i j} C_{\alpha j}$, where

$$
M=\left(\begin{array}{cccc}
1 & 1 & 1 & 1 \\
k_{\mathrm{in}, 1}^{-} & k_{\mathrm{in}, 2}^{-} & k_{\mathrm{in}, 3}^{-} & k_{\mathrm{in}, 4}^{-} \\
h_{\mathrm{in}, 1} & h_{\mathrm{in}, 2} & h_{\mathrm{in}, 3} & h_{\mathrm{in}, 4} \\
h_{\mathrm{in}, 1} k_{\mathrm{in}, 1}^{+} & h_{\mathrm{in}, 2} k_{\mathrm{in}, 2}^{+} & h_{\mathrm{in}, 3} k_{\mathrm{in}, 3}^{+} & h_{\mathrm{in}, 4} k_{\mathrm{in}, 4}^{+}
\end{array}\right),
$$

In these equations, 


$$
k_{\mathrm{in}, i}^{ \pm}=k_{\mathrm{in}, i} \pm v_{0} \quad \text { and } \quad k_{\mathrm{out}, \alpha}^{ \pm}=k_{\mathrm{out}, \alpha} \pm v_{0} / \sigma^{2} .
$$

\section{E. Boundary conditions at the sink. Complex eigenvalues}

We have already found the modes in the inner and outer regions, as well as their relation. To determine which (complex-frequency) modes will be present, it only remains to impose the boundary conditions dictated by the presence of the sink at $x=0$.

As we already mentioned, the symmetry of the system under reflection $(x \rightarrow-x)$ allows us to study only the region $x>0$, provided that we study the even and odd perturbations separately. For odd fluctuations $\left[\phi_{0}(x, \tau)=-\phi_{0}(-x, \tau)\right]$, boundary conditions (3) become

$$
\phi_{\mathrm{o}}(0, \tau)=0
$$

at all times $\tau$. This implies that the $u$ and $v$ components of $\phi$ must separately satisfy the boundary condition. Since we can have any linear combination of the two solutions that decay outside the horizon, we therefore have a $2 \times 2$ matrix constraint. The condition that a nonzero solution exists is that the determinant

$$
\operatorname{det}\left(\begin{array}{ll}
u_{\mathrm{in}, 1}(0) & u_{\mathrm{in}, 2}(0) \\
v_{\mathrm{in}, 1}(0) & v_{\mathrm{in}, 2}(0)
\end{array}\right)=0
$$

and therefore

$$
\sum_{i j} F_{1 i} F_{2 j}\left(h_{\mathrm{in}, i}-h_{\mathrm{in}, j}\right) e^{-i\left(k_{\mathrm{in}, i}+k_{\mathrm{in}, j}\right) L}=0 .
$$

For even fluctuations $\left[\phi_{\mathrm{e}}(x, \tau)=\phi_{\mathrm{e}}(-x, \tau)\right]$, boundary conditions (3) become

$$
\phi_{\mathrm{e}}^{\prime}(0, \tau)+i v_{0} \phi_{\mathrm{e}}(0, \tau)=0
$$

which implies that

$$
\sum_{i j} F_{1 i} F_{2 j}\left(h_{\mathrm{in}, i}-h_{\mathrm{in}, j}\right) k_{\mathrm{in}, i} k_{\mathrm{in}, j} e^{-i\left(k_{\mathrm{in}, i}+k_{\mathrm{in}, j}\right) L}=0 .
$$

For fixed $L, \mathcal{U}, v_{0}$, and $\sigma$, the quantities $F, h_{\text {in }}$, and $k_{\text {in }}$ that appear Eqs. (7) and (8) are only functions of $\omega$. Therefore, the solutions to these equations are all the possible complex eigenfrequencies, which depend on the free parameters that determine the model, namely, the size $2 L$ of the inner region, the speed of sound inside $c_{0}$, the relative change of the speed of sound between the inner and the outer regions $\sigma$, and the flow velocity inside $v_{0}$ (related to the characteristics of the outcoupler laser). In practice, there are also other parameters of the condensate such as its size $2 D$ (which has been made arbitrarily large) and the size of the intermediate regions $\epsilon$ (which has been made arbitrarily small).

Equations (7) and (8) can be solved numerically for different values of the parameters $\sigma, \mathcal{U}, v_{0}$, and $L$. The numerical method employed is the following. The equations above have the form

$$
f\left(\omega ; \sigma, \mathcal{U}, v_{0}, L\right)=0
$$

where $f$ and $\omega$ are both in general complex. We plot contours of constant absolute value of $f$ in the complex $\omega$ plane; where $|f|$ approaches zero, we have an eigenfrequency.

The distribution of complex solutions in the complex $\omega$ plane depends on the size of the inner region $L$, for given $\sigma$, $\mathcal{U}$, and $v_{0}$. Direct inspection of the numerical results shows that the number of instabilities increases by one when the black-hole size $L$ is increased by $\pi / k_{0}$, where $k_{0}=\sqrt{v_{0}^{2}-c_{0}^{2}}$. More explicitly, for $L$ smaller than $\pi / k_{0}-\delta$ ( $\delta$ being much smaller than $\pi / k_{0}$ ) there are no complex eigenfrequencies; for $(L+\delta) k_{0} / \pi \in[n, n+1]$, with $n=1,2, \ldots$, we have $n$ complex solutions except for $L=(n+1 / 2) \pi / k_{0}$, where we find $n-1$ complex solutions instead of $n$ [i.e., there is one mode for which $\operatorname{Im}(\omega)=0$ within numerical resolution]. This can be easily interpreted qualitatively since the unstable modes are basically the bound states in the black hole, and the highest wave number $k$ on the positive norm upper branch, for the barely bound state with $\omega \rightarrow 0^{-}$, is exactly $k_{0}$. So the threshold is simply when the well becomes large enough to have a bound state; the small $\delta$ displacement comes in because the horizon is not exactly a hard wall; the situation is similar for the extra bound state every $\pi / k_{0}$. Thus stability can only be achieved for small sizes of the inner region, $L \lesssim \pi / k_{0}$. As we discussed in Sec. II, the wavelength $2 \pi / k$ of the perturbations must be smaller than this size, which implies $k>2 \pi / L \gtrsim 2 k_{0}$. However, for these perturbations the hydrodynamic approximation, which requires $k \lesssim 2 k_{0}$, is not valid. Therefore there are no stable black-hole configurations in a strict sense. The sizes of the imaginary parts of the complex solutions decrease as the size $L$ of the interior of the black hole increases. Thus, although a larger hole has more unstable modes, it is actually less unstable (and might even became quasistable in the sense that its instability timescale would be longer than the experimental duration).

\section{CONCLUSIONS}

We have seen that dilute Bose-Einstein condensates admit, under appropriate conditions, configurations that closely ressemble gravitational black holes. We have analyzed in detail the case of a condensate in a ring trap, and proposed a realistic scheme for adiabatically creating stable sonic black/ white holes, and we have seen that there exist stable and unstable black-hole configurations. We have also studied a model for a sink-generated sonic black hole in an infinite one-dimensional condensate. The dynamical instabilities can be interpreted as coming from quasiparticle pair creation, as in the well-known suggested mechanism for black-hole evaporation. Generalizations to spherical or quasi-twodimensional traps, with flows generated by laser-driven atom sinks, should also be possible, and should behave similarly. While our analysis has been limited to Bogoliubov theory, further theoretical problems of backreaction and other corrections to simple mean-field theory should be more tractable for condensates than for other systems analogous to black holes. We expect that experiments along the lines we have 
proposed, including both creation and evaporation of sonic black holes, can be performed with state-of-the-art or planned technology.

\section{ACKNOWLEDGMENTS}

We thank the Austrian Science Foundation and the European Union TMR networks ERBFMRX-CT96-0002 and ERB-FMRX-CT96-0087. J.R.A. is grateful to Ted Jabobson for useful discussions.

\section{APPENDIX: COMPLEX FREQUENCIES. REDUNDANCY AND NORMALIZATION}

In this appendix, we will analyze the issue of the redundancy and normalization of the Bogoliubov modes in the presence of complex frequencies from a general point of view. Dynamical instabilities in quantum field theory, and the quantization of dynamically unstable modes, do not seem to be widely understood: for instance, it is common to read axiomatic statements that one must only quantize positive norm modes, even though this implicitly neglects dynamical instabilities, and does not follow in general from the fundamental commutation relations. However, some explicit treatments of quantum instabilities have been available in the literature for some time [25]; here we review this subject in the specific Bogoliubov context.

We will begin by writing the Bogoliubov equations in their most usual form,

$$
\hbar \omega_{j}\left(\begin{array}{l}
u_{j} \\
v_{j}
\end{array}\right)=\left(\begin{array}{cc}
h_{0}(\mathbf{x}) & c(\mathbf{x})^{2} e^{2 i \vartheta(\mathbf{x})} \\
-c(\mathbf{x})^{2} e^{2 i \vartheta(\mathbf{x})} & -h_{0}(\mathbf{x})
\end{array}\right)\left(\begin{array}{l}
u_{j} \\
v_{j}
\end{array}\right),
$$

where $\quad h_{0}(\mathbf{x})=-\left(\hbar^{2} / 2 m\right) \nabla^{2}+V_{\text {ext }}(\mathbf{x})+2 m c(\mathbf{x})^{2}-\mu . \quad$ In terms of these modes, the atomic second-quantized field operator has the well-known form $\hat{\Psi}(\mathbf{x}, t)=\Psi_{s}(\mathbf{x}, t)+\hat{\psi}(\mathbf{x}, t)$, with

$$
\hat{\psi}(\mathbf{x}, t)=\sum_{j}\left[\hat{a}_{j} u_{j}(\mathbf{x}) e^{-i \omega_{j} t}+\hat{a}_{j}^{\dagger} v_{j}(\mathbf{x}) * e^{i \omega_{j}^{*} t}\right]
$$

If there is a solution $\left(u_{j}, v_{j}\right)$ to Eq. (A1) with mode frequency $\omega_{j}$, then straightforward substitution shows that $\left(u_{j^{\prime}}, v_{j^{\prime}}\right)=\left(v_{j}^{*}, u_{j}^{*}\right)$ must be a solution with frequency $\omega_{j^{\prime}}=-\omega_{j}^{*}$. If we examine the contributions of these two solutions, however, we find that together they yield but a single term, of the form $\left(\hat{a}_{j}+\hat{a}_{j^{\prime}}^{\dagger}\right) e^{-i \omega t} u_{j}(\mathbf{x})+\left(\hat{a}_{j}^{\dagger}\right.$ $\left.+\hat{a}_{j^{\prime}}\right) e^{i \omega^{*} t} v_{j}^{*}(\mathbf{x})$. It is thus a quite trivial fact that the two modes $j$ and $j^{\prime}$ are redundant. We are free to simplify our notation by redefining $\hat{a}_{j}+\hat{a}_{j^{\prime}}^{\dagger} \rightarrow \hat{a}_{j}$, and eliminating mode $j^{\prime}$ (leaving it out of the sum over frequencies). Alternatively we could of course eliminate $j$ and keep $j^{\prime}$. Which of these two notational conventions we should take is best determined by the commutator $\left[\hat{a}_{j}+\hat{a}_{j^{\prime}}^{\dagger}, \hat{a}_{j}^{\dagger}+\hat{a}_{j^{\prime}}\right]$, which will tell us whether the coefficient of $u_{j}=v_{j^{\prime}}^{*}$ is properly an annihilation operator or a creation operator.
Since the only commutation relations that we are given are those of $\hat{\psi}$ and $\hat{\psi}^{\dagger}$, we must derive the orthogonality relation for solutions of Eq. (A1), and use it to invert Eq. (A2). We can use Eq. (A1) to show that

$$
\begin{gathered}
\left(\omega_{j}+\omega_{k}\right) M_{j k} \equiv \int d^{3} x\left(u_{j} v_{k}-v_{j} u_{k}\right)=0, \\
\left(\omega_{j}-\omega_{k}^{*}\right) N_{j k} \equiv\left(\omega_{j}-\omega_{k}^{*}\right) \int d^{3} x\left(u_{j} u_{k}^{*}-v_{j} v_{k}^{*}\right)=0,
\end{gathered}
$$

where in the case of infinite volume the right-hand sides are zero in the distributional sense, being infinitely rapidly oscillating boundary terms. This obviously implies that $M_{j k}$ vanishes unless $\omega_{k}=-\omega_{j}$, and $N_{j k}$ vanishes unless $\omega_{k}=\omega_{j}^{*}$. One can then show that it is always possible to take linear combinations among degenerate modes, and to eliminate redundant modes as just discussed, in such a way as to make $M_{j k}$ always vanish, and $N_{j k}=\delta_{k \bar{j}}$, where for every $j$ there is a single dual mode $\bar{J}$, with $\omega_{j}^{-}=\omega_{j}^{*}$. In the case of real $\omega_{j}$, but only then, we have $\bar{J}=j$. In general, however, duality is reciprocal (the dual mode of $\bar{J}$ is always $j$ ).

The result is that we can now insert Eq. (A2) into the second-quantized Hamiltonian, with the $T$-matrix approximation for the interparticle interaction, to obtain the linearized Bogoliubov Hamiltonian for the perturbations:

$$
\hat{H}=\hbar \sum_{j} \omega_{j} \hat{a}_{J}^{\dagger} \hat{a}_{j}
$$

Since the sum over all modes $j$ also includes the dual to every mode with complex $\omega_{j}, \quad \hat{H}$ is manifestly Hermitian, even though $\omega_{j}$ need not be real. We can also invert Eq. (A2) to learn that

$$
\hat{a}_{j}=\int d^{3} x\left[u_{J}^{*} \hat{\delta} \psi+v_{J}^{-} \hat{\delta} \psi^{\dagger}\right],
$$

which with Eq. (A3) implies the commutation relations

$$
\left[\hat{a}_{j}, \hat{a}_{k}^{\dagger}\right]=\delta_{k \bar{J}}, \quad\left[\hat{a}_{j}, \hat{a}_{k}\right]=0 .
$$

For all $j$ with real $\omega_{j}$, Eq. (A6) are merely standard canonical commutation relations; our normalization conventions $M_{j k}=0$ and $N_{j k}=\delta_{j k}$ are likewise the ones most often presented. In the case of complex $\omega_{j}$ where $\bar{J} \neq j$, however, Eq. (A6) imply that the canonical conjugate of $\hat{a}_{j}$ is $\hat{a}_{J}^{\dagger}$, and this is no longer the same as the Hermitian conjugate $\hat{a}_{j}^{\dagger}$. In fact for complex $\omega_{j}$ we have $\left[\hat{a}_{j}^{\dagger}, \hat{a}_{j}\right]=0$; this already follows from the second line of Eq. (A3), which implies that the norm $N_{j j}$ of any mode with complex $\omega_{j}$ is zero. But if $\hat{a}_{j}$ and $\hat{a}_{j}^{\dagger}$ commute, then it is clear that neither $\hat{a}_{j}$ nor $\hat{a}_{J}^{-}$is really a harmonic-oscillator annihilation operator in the usual sense, nor are $\hat{a}_{j}^{\dagger}$ or $\hat{a}_{J}^{\dagger}$ proper creation operators. The commutation relations (A6) are validly derived from the fundamental commutation relations for $\hat{\psi}$ and $\hat{\psi}^{\dagger}$; however, they do not imply, 
for instance, that either $\hat{a}_{j}^{\dagger} \hat{a}_{j}$ or $\hat{a}_{j}^{\dagger} \hat{a}_{j}$ has the discrete, equally spaced spectrum, bounded from below, that one expects of a quasiparticle number operator.

To understand the dual pairs of modes with complex frequencies, we can define the ordinary annihilation operators

$$
\hat{b}_{j}=\frac{1}{\sqrt{2}}\left(\hat{a}_{j}+\hat{a}_{J}^{-}\right), \quad \hat{b}_{J}^{-}=\frac{i}{\sqrt{2}}\left(\hat{a}_{j}^{\dagger}-\hat{a}_{J}^{\dagger}\right)
$$

and their Hermitian conjugates, among which the only nonvanishing commutators are the ordinary

$$
\left[\hat{b}_{j}, \hat{b}_{j}^{\dagger}\right]=\left[\hat{b}_{J}^{-}, \hat{b}_{J}^{\dagger}\right]=1 \text {. }
$$

In terms of these operators, which are harmonic-oscillator annihilation and creation operators with all the familiar properties of such, the $j, \bar{J}$ subsector of the Bogoliubov Hamiltonian $\hat{H}$ appears as

$$
\hat{H}_{j, \bar{J}}=\operatorname{Re}\left(\omega_{j}\right)\left[\hat{b}_{j}^{\dagger} \hat{b}_{j}-\hat{b}_{J}^{\dagger} \hat{b}_{J}^{-}\right]-\operatorname{Im}\left(\omega_{j}\right)\left[\hat{b}^{\dagger} \hat{b}_{J}^{\dagger}+\hat{b}_{j} \hat{b}_{J}^{-}\right] .
$$

Note that Eq. (A9) is only the simplest form in which one may write the $j, \bar{J}$ sector of the Hamiltonian: by introducing appropriate factors of $e^{ \pm i \alpha / 2} / \cos \alpha$ into Eq. (A7), for any $\alpha$, we can make $\operatorname{Im}\left(\omega_{j}\right) \rightarrow \operatorname{Im}\left(\omega_{j}\right) / \cos \alpha$ and add a term $\operatorname{Im}\left(\omega_{j}\right) \tan \alpha\left(\hat{b}_{j}^{\dagger} \hat{b}_{j}+\hat{b}_{J}^{\dagger} \hat{b}_{J}^{-}\right)$.

We can now examine the spectrum of $\hat{H}_{j, \bar{j}}$ by considering it in the basis of eigenstates of $\hat{n}=\hat{b}_{j}^{\dagger} \hat{b}_{j}+\hat{b}_{J}^{\dagger} \hat{b}_{J}^{-}$and $\hat{\Delta}$ $=\hat{b}_{j}^{\dagger} \hat{b}_{j}-\hat{b}_{j}^{\dagger} \hat{b}_{\bar{J}}^{-}$. In fact, $\hat{\Delta}$ commutes with $\hat{H}_{j, \bar{J}}$, so defining

$$
\begin{aligned}
& \left|E_{\Delta}\right\rangle=\sum_{n=0}^{\infty} c_{n}|n+\Delta\rangle|n\rangle \Delta \geqslant 0, \\
& \left|E_{\Delta}\right\rangle=\sum_{n=0}^{\infty} c_{n}|n\rangle|n+\Delta\rangle \Delta \leqslant 0,
\end{aligned}
$$

where $\hat{b}_{j}^{\dagger} \hat{b}_{j}|m\rangle|n\rangle=m|m\rangle|n\rangle$ and $\hat{b}_{J}^{\dagger} \hat{b}_{J}^{-}|m\rangle|n\rangle=n|m\rangle|n\rangle$; we find $\hat{H}_{j, j}\left|E_{\Delta}\right\rangle=\left[\hbar \Delta \operatorname{Re}\left(\omega_{j}\right)+E_{\Delta}\right]\left|E_{\Delta}\right\rangle$. We have the recursion relation

$$
E_{\Delta} c_{n}=\operatorname{Im}\left(\omega_{j}\right)\left[\sqrt{n(n+\Delta)} c_{n-1}+\sqrt{(n+1)(n+\Delta+1)} c_{n+1}\right]
$$

As $n \rightarrow \infty$, we have $c_{n+1} \rightarrow-c_{n-1}$, and so $\Sigma_{n}\left|c_{n}\right|^{2}$ does not converge: none of the eigenstates of $\hat{H}_{j, \bar{j}}$ is normalizable. However, one can obtain delta-function normalization for a continuous spectrum of real $E_{\Delta}$, bounded neither above nor below.

That the Hamiltonian $\hat{H}$ is unbounded from below does not indicate anything unphysical about our model: we have simply linearized about an unstable excited state of the nonlinear full Hamiltonian, which is bounded from below. Real negative frequencies $\omega_{j}$, where our convention $N_{j j}=1$ has been imposed, indicate energetic instabilities, whereby the system will decay in the presence of dissipation. Complex $\omega_{j}$, on the other hand, indicate dynamical instabilities. Classically, a dynamically unstable system will exponentially diverge from an initial stationary state if is perturbed, even without dissipation. Quantum mechanically, we have just seen that a dynamically unstable system has no normalizable stationary states. If an initially stable system is driven into a state which is stationary but dynamically unstable at the classical (mean-field) level, the initial state will have had finite Hilbert space norm, and hence under unitary evolution the final state will have the same norm. Thus it will not be a stationary state; one may say that quantum fluctuations will always trigger the dynamical instability. For a logarithmically long period of time, however, the linearized theory will still remain valid. In this sense, our linearized description of quantum dynamical instabilities is sound.
[1] M. H. Anderson, J. R. Ensher, M. R. Matthews, C. E. Wieman, and E. A. Cornell, Science 269, 198 (1995). K. B. Davis et al., Phys. Rev. Lett. 75, 3969 (1995).

[2] See, e.g., F. Dalfovo, S. Giorgini, L. P. Pitaevskii, and S. Stringari, Rev. Mod. Phys. 71, 463 (1999).

[3] For a review, see, e.g., G. E. Volovik, in Topological Defects and the Non-Equilibrium Dynamics of Symmetry Breaking Phase Transitions, edited by Y. M. Bunkov and H. Godfrin (Kluwer, Dordrecht, 2000), p. 353; V. B. Eltsov, M. Krusius, and G. E. Volovik, e-print cond-mat/9809125.

[4] W. G. Unruh, Phys. Rev. Lett. 46, 1351 (1981).

[5] W. G. Unruh, Phys. Rev. D 51, 2827 (1995).

[6] M. Visser, e-print gr-qc/9311028; S. Liberati, S. Sonego, and M. Visser, Class. Quantum Grav. 17, 2903 (2000).

[7] M. Visser, Phys. Rev. Lett. 80, 3436 (1998); Class. Quantum Grav. 15, 1767 (1998).

[8] C. W. Misner, K. S. Thorne, and J. A. Wheeler, Gravitation
(Freeman, San Francisco, 1973).

[9] S. W. Hawking, Nature (London) 248, 30 (1974); Commun. Math. Phys. 43, 199 (1975).

[10] T. Jacobson, Phys. Rev. D 44, 1731 (1991).

[11] S. Corley and T. Jacobson, Phys. Rev. D 59, 4011 (1999); S. Corley, ibid. 57, 6280 (1998).

[12] For a review, see, e.g., T. Jacobson, Prog. Theor. Phys. Suppl. 136, 1 (1999).

[13] V. M. H. Ruutu et al., Nature (London) 382, 334 (1996); T. A. Jacobson and G. E. Volovik, Phys. Rev. D 58, 4021 (1998); G. E. Volovik, Pis'ma Zh. Eksp. Teor. Fiz. 69, 662 (1999) [JETP Lett. 69, 705 (1999)].

[14] B. Reznik, e-print gr-qc/9703076.

[15] U. Leonhardt and P. Piwnicki, Phys. Rev. Lett. 84, 822 (2000); Phys. Rev. A 60, 4301 (1999).

[16] M. R. Matthews et al., Phys. Rev. Lett. 83, 2498 (1999); L. Denget et al., Nature (London) 398, 218 (1999); S. Burger 
et al., Phys. Rev. Lett. 83, 5198 (1999).

[17] L. J. Garay, J. R. Anglin, J. I. Cirac, and P. Zoller, Phys. Rev. Lett. 85, 4643 (2000).

[18] M. R. Andrews et al., Science 275, 637 (1997); I. Bloch, T. W. Hänsch, and T. Esslinger, Phys. Rev. Lett. 82, 3008 (1999); E. W. Hagley et al., Science 283, 1706 (1999).

[19] See, e.g., T. Winiecki et al., Europhys. Lett. 48, 475 (1999).

[20] See, e.g., U. Al Khawaja et al., Phys. Rev. A 60, 1507 (1999); D. L. Feder et al., ibid. 61, 00116011 (2000).

[21] Yu. Kagan, N. Prokof'ev, and B. V. Svistunov, Phys. Rev. A 61, 045601 (2000).

[22] L. J. Garay, J. R. Anglin, J. I. Cirac, and P. Zoller (unpub- lished).

[23] P. O. Fedichev and G. V. Shlyapnikov, Phys. Rev. A 60, R1779 (1999).

[24] R. Dum, J. I. Cirac, M. Lewenstein, and P. Zoller, Phys. Rev. Lett. 80, 2972 (1998); J. Williams and M. Holland, Nature (London) 401, 568 (1999).

[25] See Gungwon Kang, preprint, hep-th/9603166 for a concise pedagogical illustration, and references therein, especially $\mathrm{S}$. A. Fulling, Aspects of Quantum Field Theory in Curved Spacetime (Cambridge University Press, Cambridge, 1989); B. Schroer and J. A. Swieca, Phys. Rev. D 2, 2938 (1970). 